\title{
THE ROLE OF ERG/VEP AND EYE MOVEMENT RECORDINGS IN CHILDREN WITH OCULAR MOTOR APRAXIA
}

\author{
FATIMA S. SHAWKAT, CHRISTOPHER M. HARRIS, DAVID S. I. TAYLOR and \\ ANTHONY KRISS \\ London
}

\begin{abstract}
SUMMARY
Ocular motor apraxia (OMA) is characterised by an intermittent inability to initiate voluntary saccades, and a failure to produce optokinetic and vestibular quick phases. Some patients have no other abnormalities (idiopathic OMA), whereas in others it appears associated with a variety of neurological conditions which may affect the sensory visual pathway. Electroretinograms (ERGs), flash and pattern visual evoked potentials (VEPs) and eye movements were assessed in 53 children with OMA (age range 17 days to 14 years) to determine their efficacy in helping to distinguish between idiopathic and non-idiopathic cases. Seven patients $(13.2 \%)$ had idiopathic OMA and the remaining $46(86.8 \%)$ had other associated clinical conditions. All patients had episodes of absent quick phases ('lock up') during optokinetic nystagmus (OKN) and/or vestibular testing. Flash ERGs were abnormal in only 7 patients $(13.2 \%) ; 6$ had syndromes involving a pigmentary retinopathy (Joubert's, Bardet-Biedl, infantile Refsum's, Kearns-Sayre's), and the seventh had a cone dystrophy with vermis hypoplasia. VEPs were normal in all 7 patients with idiopathic OMA. Thirty-three $(\mathbf{7 2} \%)$ patients with OMA in association with neurological conditions had abnormal VEPs and 13 had normal VEPs $(28 \%)$. There was a significant positive correlation between VEP abnormality and poor OKN gain. VEP/ERG testing and eye movement studies are useful when OMA is suspected as they help in distinguishing isolated idiopathic cases from those with more widespread neurological abnormalities.
\end{abstract}

Cogan ${ }^{1}$ in 1952, first used the term congenital ocular motor apraxia (OMA) to describe the clinical signs of 4 patients who had difficulties in generating horizontal saccades. Since then, there have been

Correspondence to: Fatima S. Shawkat, Department of Ophthalmology, Great Ormond Street Hospital for Children, London WC1 3JH, UK. Fax: 01718298647. several reports of OMA or saccade failure occurring congenitally, with no other clinical entity. ${ }^{2-5}$ However, it can also occur as part of a wider neurological disorder: for example with structural brain abnormalities, such as agenesis of the corpus callosum ${ }^{6}$ and vermis hypoplasia; ${ }^{7}$ with neurodegenerative conditions, ${ }^{8}$ and with acquired neurological disease such as posterior fossa tumours, ${ }^{9}$ ataxia telangiectasia, ${ }^{10}$ fronto-parietal lesions, ${ }^{11,12}$ occipital cortex lesions, ${ }^{13}$ cerebellar and brainstem neoplasm ${ }^{14}$ and olivopontocerebellar degeneration. ${ }^{15,16}$ The inability to generate saccades often leads to the development of compensatory behaviour to shift direction of gaze; this includes headthrusting, blinking and tilted head posture, which enables the use of vertical eye movements that are usually unaffected. Hypometric saccades, poor optokinetic nystagmus (OKN) and contraversive deviation of the eyes on vestibular testing (due to the failure of OKN and vestibular quick phases) have also been described in OMA. ${ }^{2,17,18}$

Earlier electrophysiological studies in patients with congenital OMA reported normal flash and pattern visual evoked responses (VEPs) and electroretinograms (ERGs). ${ }^{4,13}$ However, a recent study ${ }^{20}$ found 2 of 5 children diagnosed with 'Cogan's ocular motor apraxia' to have absent ERGs but normal flash VEPs. On the basis of these findings the authors suggest the existence of two variants of the condition, one with and the other without retinal dysfunction. This prompted us to re-examine data from 53 children in whom saccade failure had been objectively identified and electrophysiological measurements had been made. Electro-oculography (EOG) and video monitoring was used to assess the eye movements. The extent of saccade failure was quantified and related to the VEP and ERG findings. 


\section{PATIENTS AND METHODS}

\section{Subjects}

Over a period of 2 years, 53 patients referred to the ophthalmology department with OMA underwent both ERG/VEP assessment and formal eye movement recording. Age at testing ranged from 17 days to 14 years, with a mean age of 3.5 years. There were 25 males and 28 females. Forty-one patients $(77.4 \%)$ were scanned (24 MRI, $17 \mathrm{CT}$ ).

Patients were divided into groups on the basis of clinical history and signs, and diagnostic findings (neuro-imaging and biochemical studies). This categorisation was carried out independent of, and prior to, the VEP/ERG assessment. Seven children had no other clinical abnormalities apart from the OMA and were labelled as having 'idiopathic' OMA. Two children had normal MRI results with other symptoms: one had early onset vertical nystagmus and microphthalmos, and the other was markedly hypotonic, developmentally delayed with gastrooesophageal reflux. There were 13 patients with non-progressive neurological deficits secondary to perinatal or postnatal problems (anoxia, ischaemia or meningitis). Thirteen children had progressive, neurodegenerative conditions (including Gaucher's disease, Krabbe's leucodystrophy, $\mathrm{GM}_{1}$ gangliosidosis, infantile Refsum's disease, Pelizaeus Merzbacher disease, propionic acidaemia) and 18 patients had structural brain anomalies (including agenesis of the corpus callosum, vermis cyst, vermis hypoplasia in isolation, and in association with Joubert's syndrome, Dandy Walker malformation, hydrocephalus, hemimegalencephaly, porencephalic cyst).

Neuro-radiological examination revealed a wide range of abnormalities the most common of which were brainstem abnormalities, cerebellar abnormalities (particularly those involving the vermis), delayed or absent myelination, and agenesis of the corpus callosum. Patients with idiopathic OMA had normal scans.

\section{Eye Movements}

Horizontal eye movements were recorded using dc EOG. Silver/silver chloride electrodes were attached with tape at the outer canthi of either eye with a reference electrode at the mid-forehead. Older patients sat alone in a Bárány chair; younger patients sat on a parent's lap. The head was restrained by a head rest, or was held by the parent. Video monitoring of the patient was carried out through the entire recording session. Infra-red monitoring was used when testing the vestibuloocular reflex (VOR) in absolute darkness.

OKN was elicited by rotating a brightly coloured and patterned, full-field curtain around the patient. Leftward and rightward OKN at speeds of 25 and 50 $\mathrm{deg} / \mathrm{s}$ were recorded. Gain of OKN (slope of the slow phase) was difficult to assess accurately because of difficulties in eliciting saccades for calibration. However, it was clearly evident that in certain cases OKN was of extremely low gain or absent when compared with control subjects recorded in our laboratory. ${ }^{19}$ Thus, OKN gain abnormalities were qualitatively ordinally scaled into $0=$ normal, $1=$ abnormal. An abnormal OKN score of 1 was given to those with conspicuously reduced gain at $25 \mathrm{deg} / \mathrm{s}$ stimulus speeds or when OKN was totally absent.

VOR was tested by rotating the patient on the Bárány chair while in complete darkness. The chair was accelerated at $18 \mathrm{deg} / \mathrm{s}^{2}$ to a speed of $80 \mathrm{deg} / \mathrm{s}$ and this speed was maintained for 40 seconds before decelerating at $18 \mathrm{deg} / \mathrm{s}^{2}$ to rest. After another 40 seconds at rest, the chair was rotated in the opposite direction. Per- and post-rotatory vestibular nystagmus were recorded in both directions.

\section{Electrophysiology}

VEPs were recorded using silver/silver chloride EEG electrodes placed in a line across the occipital scalp. A midline electrode was sited at $\mathrm{O}_{\mathrm{z}}$ (10-20 system, approximately $3 \mathrm{~cm}$ above the inion) and lateral electrodes were placed midway between the $\mathrm{O}_{z}$ electrode and the ear. All occipital electrodes were referred to a common frontal electrode at $F_{z}(10-20$ system). The electroretinogram (ERG) was recorded from a lower eyelid skin electrode also referred to $F_{z}$. Full-field pattern reversal VEPs were elicited using a checkerboard pattern presented on a large TV display (subtending 28 degrees horizontal by 21 degrees vertical). Stimulus check-sizes ranged from 25 minutes to 7 degrees. A Grass PS22 photic stimulator, held $15 \mathrm{~cm}$ in front of the eye, was used to elicit flash VEPs and mixed cone/rod ERGs under fully darkened laboratory conditions.

\section{RESULTS}

\section{Eye Movements}

Clinical examination identified head thrusting behaviour in 30 of the patients $(57 \%)$. The remaining 23 (43\%) did not head thrust and OMA was identified during the formal eye movement recording. The majority of patients with idiopathic OMA (86\%) showed head thrusting behaviour; in contrast, it was evident in only $10 \%$ of patients with associated neurodegenerative conditions. There was no obvious trend in children with structural malformations of the central nervous system (CNS), of whom 56\% exhibited head thrusting, whereas fewer $(30 \%)$ patients with peri- or postnatal problems showed this compensatory behaviour.

Saccade failure during OKN and VOR testing was identified as episodes of absent quick phases (Fig. 1). When quick phases at the end of an OKN or vestibular nystagmus slow phase are absent the eyes 
A. OKN to Leftwand Curtain Rotation

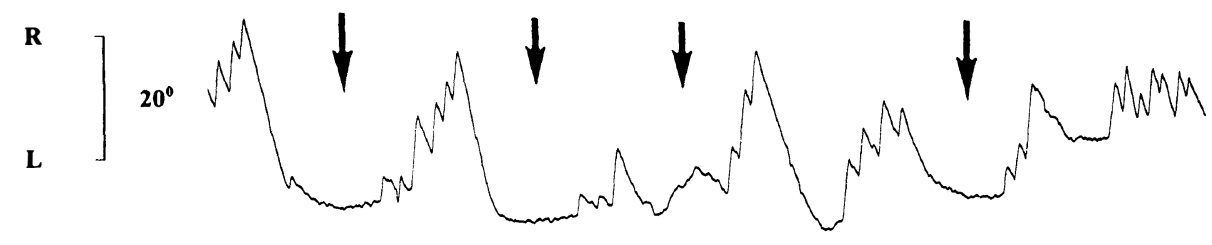

B. VOR to Rightwand Chair Rotation

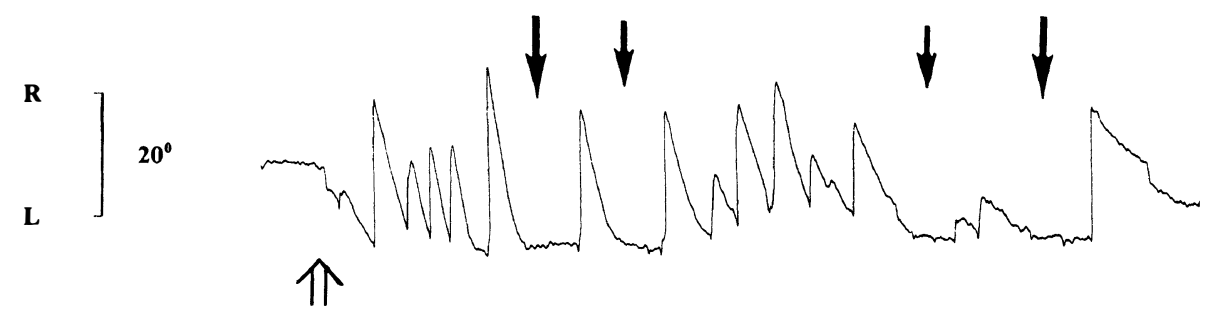

Fig. 1. EOG traces of optokinetic nystagmus $(A)$ elicited by leftward curtain rotation at a speed of $25 \mathrm{deg} / \mathrm{s}$, and vestibular nystagmus $(B)$ elicited by chair rotation (open arrow) at $80 \mathrm{deg} / \mathrm{s}$. Episodes of 'lock up' (filled arrows), where there is failure in generating quick phases, can be clearly discerned during both tests.

remain in extreme lateral deviation in the direction of curtain rotation, or in the opposite direction to chair rotation. We call this phenomenon 'lock up', and this provides a measure of the severity of saccade failure. It is measured by the percentage of time the eyes remained in extreme lateral deviation during OKN testing at the two speeds (25 and 50 $\mathrm{deg} / \mathrm{s}$ ) and during VOR testing. Measures of lock up are therefore relative and independent of calibration. Saccade failure was usually intermittent and normal quick phases were detected in the majority of patients. All patients who locked up during OKN testing, invariably locked up during VOR testing. There were no significant differences (ANOVA, $p<0.5)$ in the extent of lock up between idiopathic OMA patients and patients with other associated neurological problems.

OKN was also evaluated by assessing the gain of the slow phases at $25 \mathrm{deg} / \mathrm{s}$ stimulus speed. Eightyone per cent of cases had marked OKN abnormalities. All patients with idiopathic "congenital ocular motor apraxia' had normal OKN gain, but there was no statistical difference (Kruskal-Wallis, $p<0.5$ ) between those with idiopathic OMA and those with OMA in association with other conditions.

\section{Electrophysiology}

ERGs were not detectable or significantly attenuated in 7 patients. Six of these patients with markedly attenuated scotopic and photopic ERGs had a diagnosis in which there was a pigmentary retinopathy: 3 with Joubert's, 1 with Bardet-Biedl, 1 with infantile Refsum's, and 1 with Kearns-Sayre's syndrome. The seventh patient had attenuated ERGs to bright white and red flash, but ERGs were preserved to dim blue flash, indicating retinal dysfunction primarily affecting the cones.

Flash and pattern VEPs were compared with ageappropriate laboratory norms. Thirty-eight patients ( $72 \%$ ) had abnormal VEPs (that is attenuated and/or delayed). In 8 patients latencies were significantly prolonged $(>2.5 \mathrm{SD})$ and in the remainder VEPs were significantly attenuated $(<2.5 \mathrm{SD})$. The seven cases with idiopathic OMA had normal VEPs (mean for $50^{\prime}$ checks $=16.7 \mu \mathrm{V}$ at $106 \mathrm{~ms}$; mean for flash $=$ $24.1 \mu \mathrm{V}$ at $102 \mathrm{~ms}$ ).

Patients with neurological conditions could be divided into three main groups: (1) 13 patients had peri/postnatal problems, of whom $80 \%$ had abnormal VEPs; (2) 18 patients had CNS structural anomalies, of whom $77 \%$ had abnormal VEPs; and (3) 13 patients had progressive neurodegenerative disease, of whom $85 \%$ had abnormal VEPs. There were no conspicuous differences in VEP amplitude deficits between these general diagnostic categories; however, VEP latencies were particularly prolonged in the neurodegenerative group of patients. This was particularly marked for VEPs to pattern reversal stimulation (Fig. 2). It can be seen from Fig. 2 that pattern VEPs in the neurodegenerative patient group are delayed when compared with patients with idiopathic OMA, or OMA associated with CNS structural malformations. Children with peri/post- 


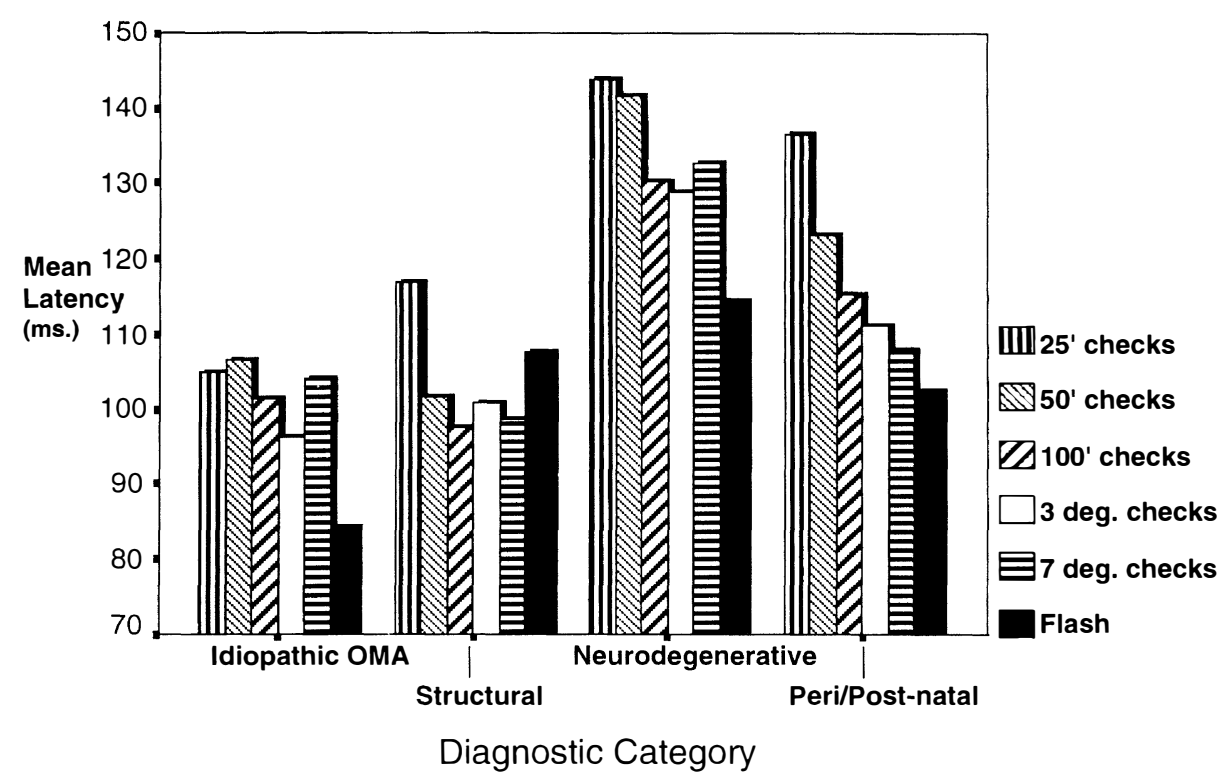

Fig. 2. Bar chart of mean pattern and flash VEP latencies for the different diagnostic categories. It can be seen that patients with idiopathic OMA have latencies within the normal range, whereas those with neurodegenerative conditions have the most prolonged latencies, particularly when the smaller check-sizes are used.

natal problems tended to have prolonged VEPs to the smaller check-sizes.

Table I compares the mean amplitudes and latencies for our laboratory controls with those of the 7 patients with idiopathic OMA, the 38 patients with abnormal VEPs, and the two sub-groups of abnormal latencies and amplitudes. Patients with idiopathic OMA had VEPs within the normal range.

Fig. 3 shows the ERGs and VEPs to flash and pattern $\left(50^{\prime}\right.$ checks) stimulation of a patient with idiopathic OMA, a patient who had suffered perinatal hypoxia, a patient with a progressive neurodegenerative condition (propionic acidaemia) and a child with Joubert's syndrome and retinal dysfunction.

\section{Eye Movement and Electrophysiological Correlates}

There were no significant correlations between severity of saccade failure (lock up) during OKN and VOR testing and either VEP amplitude or latency.

The occurrence of head thrusting in an OMA patient showed a significant positive correlation with the occurrence of an abnormal ERG (Spearman's $r=0.305, p<0.04)$ and a negative correlation, that was just beyond the levels of significance, with the occurrence of a VEP abnormality $(r=-0.274$, $p=0.057$. However, analysis of individual VEP parameters showed a significant negative correlation between head thrusting and VEP amplitudes to the smallest $25^{\prime}$ checks $(r=-0.422, p<0.004)$. This negative correlation implies that head thrusting was less likely to be present in patients with wellpreserved VEPs to the small checks. However, head thrusting is more likely to occur in patients with abnormal ERGs.

OKN gain abnormality showed a positive correlation with the occurrence of abnormal VEPs (Spearman's $r=0.385, p<0.005)$. When VEP mea-

Table I. Mean VEP amplitudes and latencies for control subjects, idiopathic OMA patients, OMA patients with general VEP abnormalities and OMA patients divided into those with amplitude and latency abnormalities

\begin{tabular}{|c|c|c|c|c|c|c|c|}
\hline \multirow[b]{2}{*}{$25^{\prime}$ checks } & Control subjects & Idiopathic OMA & \multicolumn{2}{|c|}{$\begin{array}{l}\text { OMA and general } \\
\text { abnormal VEPs }\end{array}$} & \multicolumn{2}{|c|}{$\begin{array}{l}\text { OMA and } \\
\text { degraded VEPs }\end{array}$} & $\begin{array}{l}\text { OMA and } \\
\text { delayed VEPs }\end{array}$ \\
\hline & $15.2 \mu \mathrm{V} \quad \pm 10.7$ & $11.3 \mu \mathrm{V} \pm 5.8$ & $6.9 \mu \mathrm{V}$ & \pm 6.4 & $5.5 \mu \mathrm{V}$ & \pm 6.4 & $8.3 \mu \mathrm{V} \pm 8.3$ \\
\hline & $106.9 \mathrm{~ms} \pm 6.1$ & $109.5 \mathrm{~ms} \pm 7.2$ & $124.6 \mathrm{~ms}$ & \pm 22.9 & $107.9 \mathrm{~ms}$ & \pm 11.6 & $141.3 \mathrm{~ms} \quad \pm 18.5$ \\
\hline \multirow{2}{*}{$50^{\prime}$ checks } & $17.6 \mu \mathrm{V} \quad \pm 11.1$ & $16.7 \mu \mathrm{V} \pm 9.6$ & $6.5 \mu \mathrm{V}$ & \pm 5.9 & $5.2 \mu \mathrm{V}$ & \pm 3.5 & $11.3 \mu \mathrm{V} \pm 9.6$ \\
\hline & $103.3 \mathrm{~ms} \pm 4.6$ & $105.9 \mathrm{~ms} \pm 4.6$ & $119.3 \mathrm{~ms}$ & \pm 19.3 & $112.5 \mathrm{~ms}$ & \pm 14.9 & $142.2 \mathrm{~ms} \quad \pm 14.2$ \\
\hline \multirow[t]{2}{*}{$100^{\prime}$ checks } & $14.6 \mu \mathrm{V} \pm 8.0$ & $11.8 \mu \mathrm{V} \pm 6.2$ & $6.2 \mu \mathrm{V}$ & \pm 5.6 & $5.1 \mu \mathrm{V}$ & \pm 4.5 & $8.8 \mu \mathrm{V} \pm 7.2$ \\
\hline & $105.7 \mathrm{~ms} \quad \pm 4.4$ & $103.2 \mathrm{~ms} \quad \pm 10.1$ & $117.7 \mathrm{~ms}$ & \pm 19.5 & $110.3 \mathrm{~ms}$ & \pm 16.6 & $135.2 \mathrm{~ms} \quad \pm 14.1$ \\
\hline \multirow[t]{2}{*}{$3^{\circ}$ checks } & $11.6 \mu \mathrm{V} \pm 6.8$ & $8.1 \mu \mathrm{V} \pm 4.6$ & $6.9 \mu \mathrm{V}$ & \pm 5.7 & $5.3 \mu \mathrm{V}$ & \pm 3.4 & $11.5 \mu \mathrm{V} \pm 8.2$ \\
\hline & $101.1 \mathrm{~ms} \pm 5.6$ & $102.1 \mathrm{~ms} \quad \pm 8.1$ & $109.4 \mathrm{~ms}$ & \pm 22.4 & $108.9 \mathrm{~ms}$ & \pm 11.3 & $127.2 \mathrm{~ms} \quad \pm 15.1$ \\
\hline \multirow[t]{2}{*}{$7^{\circ}$ checks } & $10.7 \mu \mathrm{V} \pm 6.7$ & $8.0 \mu \mathrm{V} \pm 23$ & $6.0 \mu \mathrm{V}$ & \pm 5.2 & $4.4 \mu \mathrm{V}$ & \pm 3.3 & $9.8 \mu \mathrm{V} \pm 7.2$ \\
\hline & $101.1 \mathrm{~ms} \pm 6.5$ & $106.7 \mathrm{~ms} \pm 5.0$ & $111.7 \mathrm{~ms}$ & \pm 25.3 & $104.3 \mathrm{~ms}$ & \pm 25.5 & $129.4 \mathrm{~ms} \pm 14.5$ \\
\hline \multirow[t]{2}{*}{ Flash VEP } & $29.5 \mu \mathrm{V} \pm 13.5$ & $24.1 \mu \mathrm{V} \pm 11.6$ & $16.1 \mu \mathrm{V}$ & \pm 19.3 & $17.3 \mu \mathrm{V}$ & \pm 21.5 & $11.6 \mu \mathrm{V} \pm 6.5$ \\
\hline & $90.4 \mathrm{~ms} \pm 8.3$ & $102.0 \mathrm{~ms} \pm 22.3$ & $103.1 \mathrm{~ms}$ & \pm 24 & $98.2 \mathrm{~ms}$ & \pm 23.7 & $120.3 \mathrm{~ms} \quad \pm 16.7$ \\
\hline
\end{tabular}


A. Idiopathic OMA

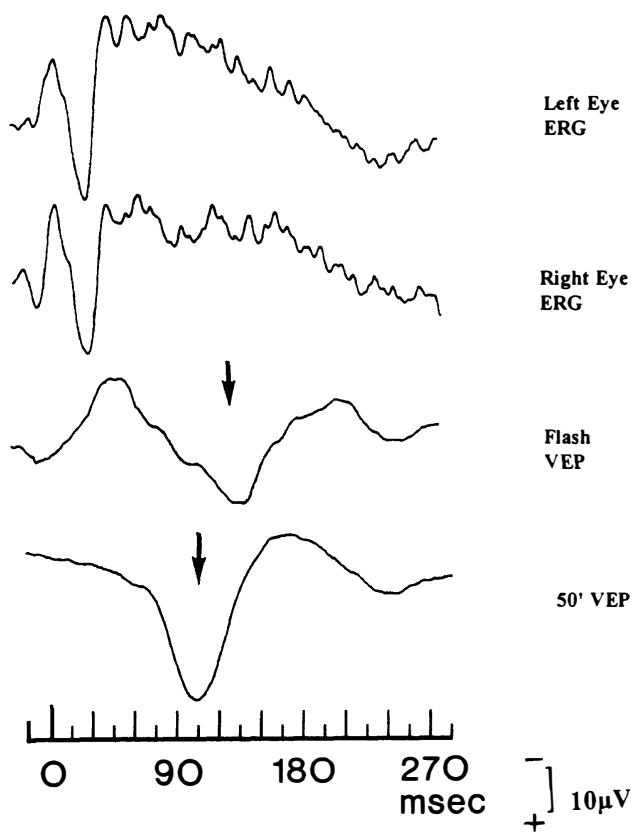

C. Neurodegenerative

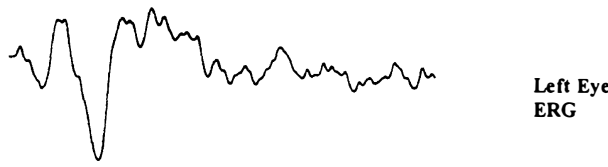
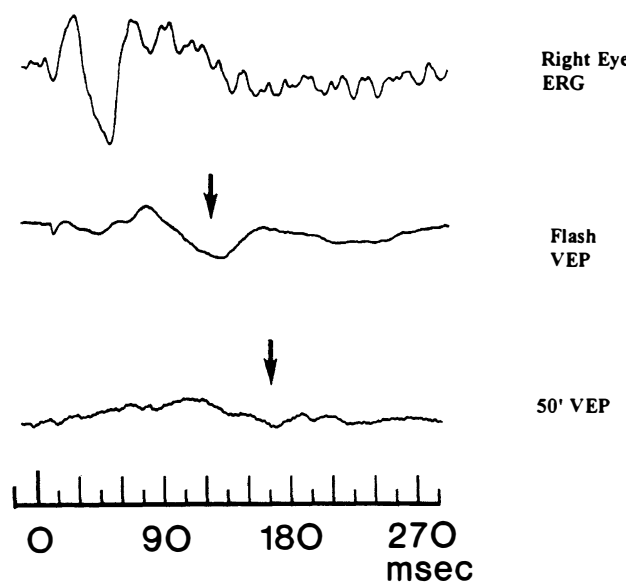

50' VEP
B. Perinatal Anoxia
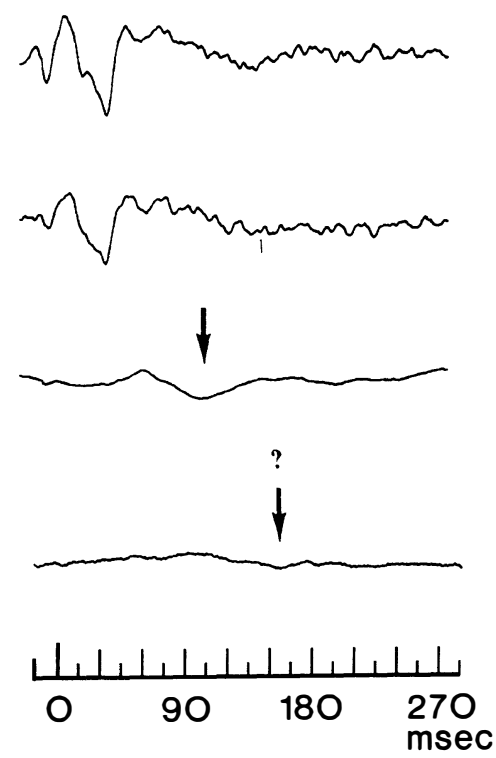

D. Joubert Syndrome

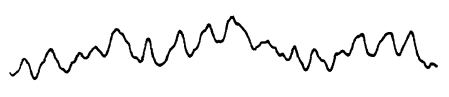

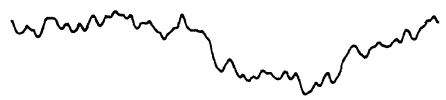

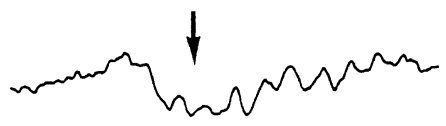

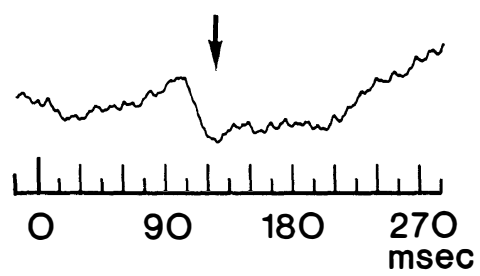

Fig. 3. Left and right eye ERGs and binocular flash and 50' pattern VEPs from a patient with idiopathic OMA $(A)$, a patient who had perinatal anoxia $(B)$, a patient with propionic acidaemia from the neurodegenerative group and a patient with Joubert's syndrome (D).

sures were analysed separately, positive significant correlations were found between OKN gain deficits and the latencies of VEPs to the three smaller checks: $25^{\prime} \quad(r=0.396, \quad p<0.004), \quad 50^{\prime} \quad(r=0.379$, $p<0.008)$ and $100^{\prime}$ checks $(r=0.301, p<0.03)$. Marked OKN abnormality was associated with more marked delays in VEPs to the moderate and small checks. A significant negative correlation was present between the amplitude of flash VEPs and
OKN deficits $(r=-0.283, p<0.05)$ : greater OKN deficits are likely to be found in patients with attenuated flash VEPs.

\section{DISCUSSION}

Ocular motor apraxia, or intermittent saccade failure, is a clinical sign found in a wide variety of clinical disorders. The occurrence of saccade failure with such a diverse number of conditions suggests 
that the system for saccade initiation is particularly sensitive to neurological disease. Our data suggest that OMA appears to be a more common symptom than previously reported in the literature. It may be subtle and detectable only with eye movement testing (head thrusting was clinically recognised only in $57 \%$ of our patients). Formal testing of eye movements is valuable in detecting OMA in infants who have not yet achieved adequate head control, as well as in those who are hypotonic and developmentally delayed and do not exhibit head thrusting compensatory behaviour. These results suggest that patients with idiopathic OMA have an isolated problem involving the generation of fast eye movements.

Our results do not support those of Magni et al., ${ }^{20}$ who stated that there is a variant of 'congenital ocular motor apraxia' which involves retinal abnormalities. All our patients with poor ERGs and OMA were diagnosed with syndromes associated with pigmentary retinopathy: Joubert's, ${ }^{7}$ Bardet-Biedl,21,22 Kearns-Sayre's ${ }^{23}$ and infantile Refsum's. ${ }^{24}$ One child had cone dysfunction and vermis hypoplasia and did not fit into a known syndrome. Magni et al.' ${ }^{20}$ conclusions were derived from 2 patients with OMA and absent ERGs, neither of whom had idiopathic saccade failure. The first was a 6-year-old with cryptorchidism, bimanual dyspraxia, developmental delay and a normal fundus and CT scan; the second was a 7-year-old with convulsions in the first year of life, hypodiadochokinesia, dysmetria, poor equilibrium and possible ataxia with salt-and-pepper mottling of the retinal pigment epithelium and frontal ventricular enlargement on CT. Such diverse symptoms may be due to as yet unrecognised conditions that involve retinal abnormalities.

OMA is a clinical sign, which may occur congenitally and not in association with any other ophthalmological or neurological abnormality. Our results support those of others ${ }^{4,13}$ showing that VEPs and ERGs are normal when OMA is idiopathic. If the VEP and/or the ERG is abnormal, then the diagnosis is not likely to be idiopathic OMA. For instance, retinal dystrophy with abnormal ERGs, OMA, possible developmenal delay and nystagmus in association with relatively well-preserved VEPs, suggests the diagnosis of Joubert's syndrome. Neuro-radiological investigations will verify the diagnosis with the demonstration of cerebellar vermis hypoplasia. OMA with retinal dystrophy has also been described in Bardet-Biedl syndrome together with other signs characteristic of the condition, that is polydactyly, obesity, mental retardation and hypogonadism. ${ }^{22}$ Absent or attenuated ERGs, associated with pigmentary retinopathy, have also been described in infantile Refsum's and
Kearns-Sayre's syndromes, though they have not previously been reported in connection with intermittent saccade failure. In infantile Refsum's disease there is a disorder of phytanic acid storage which leads to general brain atrophy and severe cerebellar granular layer hypoplasia. ${ }^{25}$ In Kearns-Sayre's syndrome there is a mitochondrial cytopathy, associated with retinal changes and chronic progressive external ophthalmoplegia together with cardiomyopathy. Reduced saccadic velocities have been reported in these patients. ${ }^{26}$ It is possible that in a severe case, saccade generation may be impaired due either to the abnormalities in the extraocular muscles themselves (increased number of sarcolemmal mitochondria $^{27}$ ) or, more likely, to CNS abnormalities - cerebellar ataxia, calcification of basal ganglia and spongy degeneration in the brain have all been reported in this condition. 28.29

Attenuated and degraded VEPs were a common feature in our patients with non-idiopathic OMA. Most patients who had peri- or postnatal problems had abnormal VEPs. Abnormal VEPs have been reported previously in infants with a variety of perinatal problems and have been found to correlate with the long-term visual outcome of the child. ${ }^{30-32}$ VEPs have been used to monitor disease progression and assess the extent of, or lack of, maturational changes. VEP abnormalities have been described in Krabbe's leucodystrophy, ${ }^{33}$ Gaucher's disease ${ }^{34}$ and Pelizaeus Merzbacher, ${ }^{35}$ as well as in children with hydrocephalus, ${ }^{36-38}$ which were all conditions associated with OMA. Several of these conditions, such as Pelizaeus Merzbacher and the leucodystrophies, have markedly abnormal or non-detectable myelination, and this is reflected in the very prolonged latencies that we found in this group of patients.

Our result showed that patients with abnormal VEPs are less likely to use head thrusting to shift direction of gaze, in contrast to those with abnormal ERGs. It is possible that children with abnormal VEPs are more likely to have extensive neurological deficits leading to poor head control. This is supported by the fact that the majority of patients with neurodegenerative conditions $(90 \%)$ did not head thrust, whereas this was common in children with idiopathic OMA (86\%).

Abnormalities in the optokinetic system would affect the OKN gain. Lesions of the posterior cortical areas and underlying white matter, ${ }^{39-41}$ as well as large lesions such as hemidecortication ${ }^{42,43}$ can result in reduced gain OKN. Decreased slow phase velocity can be due to lesions in the parietal lobe, brainstem or cerebellum. ${ }^{44}$ Reports of adults with low gain OKN and smooth pursuit in one direction have isolated the lesion to the ipsilateral parietal lobe. .5,46 $^{45}$ Our results showed that OKN deficits correlated positively with pattern VEP latencies to the smaller 
check-sizes, and negatively with VEP flash amplitudes. Prolonged latencies and attenuated flash VEPs were found in patients with OMA in association with neurological conditions and in particular with neurodegenerative and acquired diseases. In such patients, global brain abnormalities are common and likely to involve parietal cortex, brainstem and cerebellum. Thus, this association between VEP and $\mathrm{OKN}$ abnormalities can be expected. However, these results do not necessarily imply that OMA has a cortical origin.

This study has demonstrated the value of electrophysiological and eye movement testing in separating patients with idiopathic OMA from those with OMA as part of a wider and more severe neurological condition. We found no evidence of an idiopathic OMA variant with retinal involvement; if ERGs or VEPs are abnormal, then further investigation is needed to identify the underlying cause. Various neurodegenerative diseases, perinatal insults and cerebellar and brainstem anomalies appear to predispose the patient to intermittent saccade failure. This may not be easily detectable without OKN or vestibular testing. Eye movement examination and ERG/VEP testing are complementary noninvasive investigations which together give valuable information for diagnosis.

We wish to thank the Iris Fund for their support.

Key words: ERG, Eye movements, Ocular motor apraxia, VEP.

\section{REFERENCES}

1. Cogan DG. A type of ocular motor apraxia presenting jerky head movements. Trans Am J Ophthalmol Otolaryngol 1952;56:853-62.

2. Zee DS, Yee RD, Singer HS. Congenital ocular motor apraxia. Brain 1977;100:581-99.

3. Godel V, Nemet P, Lazar M. Congenital ocular motor apraxia: familial occurrence. Ophthalmologica 1979; 179:90-3.

4. Gittinger JW, Sokol S. The visual evoked potential in the diagnosis of congenital ocular motor apraxia. Am J Ophthalmol 1982;93:700-3.

5. Rosenberg ML, Wilson E. Congenital ocular motor apraxia without head thrusts. J Clin Neuro-ophthalmol 1987;7:26-8.

6. Orrison WW, Robertson WC. Congenital ocular motor apraxia: a possible disconnection syndrome. Arch Neurol 1979;36:29-31.

7. Lambert SR, Kriss A, Gresty M, et al. Joubert syndrome. Arch Ophthalmol 1989;107:709-13.

8. Cogan DB, Chu FC, Reingold D, et al. Ocular motor signs in some metabolic diseases. Arch Ophthalmol 1981;99:1802-8.

9. Lyle DJ. A discussion of ocular motor apraxia with a case presentation. Trans Am Ophthalmol Soc 1961; 59:274-85.

10. Stell R, Bronstein AM, Plant GT, et al. Ataxia telangiectasia: a reappraisal of the ocular motor features and their value in the diagnosis of atypical cases. Mov Disord 1989;4:320-9.
11. Holmes G. Spasm of fixation. Trans Ophthalmol Soc UK 1930;50:253-62.

12. Hecaen H, de Ajuriaguerra J. Balint's syndrome (psychic paralysis of visual fixation). Brain 1954; 77:373-400.

13. Reed HR, Israels S. Congenital ocular motor apraxia: a form of horizontal gaze palsy. $\mathrm{Br} \mathrm{J}$ Ophthalmol 1956;40:444-8.

14. Zaret CR, Behrens MM, Eggers HM. Congenital ocular motor apraxia and brainstem tumour. Arch Ophthalmol 1980;98:327-30.

15. Moe PC. Ocular motor apraxia in three families. Neurology 1971;221:451.

16. Aicardi J, Barbosa C, Andermann E, et al. Ataxiaocular motor apraxia: a syndrome mimicking ataxiatelangiectasia. Ann Neurol 1988;24:497-502.

17. Zee DS, Chu FC, Keigh RJ, et al. Blink-saccade synkinesis. Neurology 1983;33:1233-6.

18. Shawkat FS, Harris CM, Russell-Eggitt I, et al. Oculomotor and neuroradiological correlates in congenital saccade palsy (ocular motor apraxia). Invest Ophthalmol Vis Sci 1994;35:2034.

19. Harris CM, Jacobs M, Taylor D. The development of bi-ocular and monocular optokinetic gain from 1 to 7 months. Invest Ophthalmol Vis Sci 1994;35:2655.

20. Magni RL, Spadea A, Pece A, et al. Electroretinographic findings in congenital oculomotor apraxia (Cogan's syndrome). Doc Ophthalmol 1994;86:259-66.

21. Campo RV, Aaberg TM. Ocular and systemic manifestations of the Bardet-Biedl syndrome. Am J Ophthalmol 1982;94:750-6.

22. Lavy T, Harris CM, Shawkat FS, et al. An ERG, VEP, and eye movement assessment of children with BardetBiedl syndrome. In: Lennerstrand $\mathrm{G}$, editor. Update on strabismus and pediatric ophthalmology. Boca Raton, FL: CRC Press, 1995:549-52.

23. Kearns TP, Sayre GP. Retinitis pigmentosa, external ophthalmoplegia and complete heart block: unusual syndrome with histologic study in one of 2 cases. Arch Ophthalmol 1958;60:280-9.

24. Weleber RG, Tongue AC, Kenneway NG, et al. Ophthalmic manifestations of infantile phytanic acid storage disease. Arch Ophthalmol 1984;102:1317-21.

25. Moser HW. Peroxisomal disease. Adv Pediatr 1989; 36:1-38.

26. Metz HS, Meshel L. Ocular saccades in progressive external ophthalmoplegia. Ann Ophthalmol 1974; $6: 623-8$

27. Ringel SP, Wilson WB, Barden MT. Extraocular muscle biopsy in chronic progressive external ophthalmoplegia. Ann Neurol 1979;6:326-39.

28. Leigh RJ, Zee DS. The neurology of eye movements, 2nd ed. Philadelphia: FA Davis, 1991.

29. Daroff RB, Solitaire GB, Pincus JH, et al. Spongioform encephalopathy with chronic progressive external ophthalmoplegia: central ophthalmoplegia mimicking ocular myopathy. Neurology 1966;16:161-9.

30. Hrbek A, Karlberg P, Kjellmer I, et al. Clinical application of evoked electroencephalographic responses in new-born infants. I. Perinatal asphyxia Dev Med Child Neurol 1977;19:34-44.

31. Hakamada S, Watanabe K, Hara K, et al. The evolution of visual and auditory evoked potentials in infants with perinatal disorder. Brain Dev 1981; 3:339-44.

32. Taylor MJ, Murphy WJ, Whyte HE. Prognostic reliability of SEPs and VEPs in asphyxiated term infants. Dev Med Child Neurol 1992;34:507-15.

33. Taylor MJ. Evoked potentials in paediatrics. In: 
Halliday AM, editor. Evoked potentials in clinical testing, 2nd ed. London: Churchill Livingstone, 1993: 489-521.

34. Vivian AJ, Harris CM, Kriss A, et al. Oculomotor signs in infantile Gaucher disease. Neuroophthalmol 1993; 13:151-5.

35. Markand ON, Garg BP, DeMeyer WE, et al. Brainstem auditory, visual and somatosensory evoked potentials in leukodystrophies. Electroencephalogr Clin Neurophysiol 1982;54:39-48.

36. Ehle AL, Sklar F. Visual evoked potentials in infants with hydrocephalus. Neurology 1979;29:1541-4.

37. Guthkelch AM, Sclabassi RJ, Vries JK. Changes in the visual evoked potentials of hydrocephalic children. Neurosurgery 1982;11:599-602.

38. Watanabe K, Yamada H, Hara K, et al. Neurophysiological evaluation of newborns with congenital hydrocephalus. Clin Electroencephalogr 1984;15:22-31.

39. Thurston SE, Leigh RJ, Crawford T, et al. Two distinct deficits of visual tracking caused by unilateral lesions of cerebral cortex in humans. Ann Neurol 1988;23: 266-73.

40. Morrow MJ, Sharpe JA. Cerebral hemispheric localisation of smooth pursuit asymmetry. Neurology 1990;40:284-92.
41. Jacobs M, Shawkat F, Harris CM, et al. Eye movements and electrophysiological findings in an infant with hemicerebral pathology. Dev Med Child Neurol 1993;35:431-5.

42. Troost BT, Daroff RB, Weber RB, Dell'Osso LF Hemispheric control of eye movements. II. Quantitative analysis of smooth pursuit in a hemispherectomy patient. Arch Neurol 1972;27:449-52.

43. Sharpe JA, Lo AW. Voluntary and visual control of the vestibuloocular reflex after cerebral hemidecortication. Ann Neurol 1979;10:164-72.

44. Baloh RW, Sills A, Honrubia V. Eye tracking and optokinetic nystagmus: results of quantitative testing in patients with well-defined nervous system lesions. Ann Otol Rhinol Laryngol 1977;86:108-14.

45. Kjallman L, Frisén L. The cerebral ocular pursuit pathways: a clinicoradiological study of small-field optokinetic nystagmus. J Clin Neuro-Ophthalmol 1986;6:209-14.

46. Heide W, Koenig E, Dichgans J. Optokinetic nystagmus, self-motion sensation and their after-effects in patients with occipito-parietal lesions. Clin Vis Sci 1990;5:145-56. 